\title{
THE NUMERICAL SOLUTION OF NONLINEAR OPTIMAL CONTROL PROBLEMS BY USING OPERATIONAL MATRIX OF BERNSTEIN POLYNOMIALS
}

\author{
NAJMEH GHADERI* AND MOHAMMAD HADI FARAHI
}

\begin{abstract}
A numerical approach based on Bernstein polynomials basis is presented to unravel optimal control of nonlinear systems. The operational matrices of differentiation, integration, and product are introduced. Then, these matrices are implemented to decrease the solution of the nonlinear optimal control problem to the solution of the quadratic programming problem which can be solved with many algorithms and softwares. This method is easy to implement with an accurate solution. Some examples are included to demonstrate the validity and applicability of the technique.
\end{abstract}

MSC(2010): 49J15; 33C47; 90C20.

Keywords: Optimal control of nonlinear systems, Operational matrices, Quadratic programming problem, Bernstein polynomials.

\section{Introduction and Background}

One of the most challenging and difficult issues in control theory is the nonlinear optimal control problems (NOCPs) $([3,13])$. Many methods have been applied to solve optimal control of nonlinear systems, but these methods can solve a few classes of nonlinear systems. For instance, Lee and Chang [15] studied a special class of optimal control of nonlinear systems by employing general orthogonal polynomials. Vlassenbroock and Dooren used Chebyshev polynomials for solving the NOCPs ([22]). Next, a general technique was grown by presenting Block Plus functions for solving the NOCPs in [21]. Besides, in [2], first the NOCPs transformed to the calculus of variations problem, then the new problem was solved with discretization approach. By using Quasilinearisation and Chebyshev polynomials, an approximation technique was implemented to solve the NOCPs in [11]. Mohan and Kar applied Block Plus functions and Legendre polynomials to solve the NOCPs ([17]). Also, using Block Plus functions and Bernoulli polynomials, NOCPs was solved in [16].

Date: Received: January 19, 2021, Accepted: April 3, 2021.

${ }^{*}$ Corresponding author. 
In recent years, Bernstein polynomials (BPs) and orthogonal functions have been applied to evaluate various problems. For example, in [7] and [25], BPs were employed to solve the partial differential equations and fractional optimal control problems. Furthermore, they were used for solving optimal control of time-varying singular systems and the nonlinear age-structured population models $[5,26]$. Bhatti [6], presented an approximation technique to a fractional differential equation by employing fractional BPs matrices. Additionally, these polynomials were exploited to solve optimal control systems with constant and Pantograph delays [12]. The cubic B-spline functions and Bernstein multi-scaling functions were employed in [9] to solve an inverse heat condition problem along with the Ritz-Galerkin method. In [20], a Legendre operational matrices were used to unravel a class of fractional differential equations. Exerting Taylor series as well as Jacobi polynomials, operational matrices of Jacobi orthogonal functions were obtained [14, 10].

In the present study, we consider a wide class of NOCPs which most of them do not have exact solutions, so finding the numerical solutions to these problems is very useful. Besides, these problems are very interesting to engineers and mathematicians, since most real-world systems in science and engineering are inherently nonlinear in nature. Using the Bernstein operational matrices, we obtain a computationally simple and efficient approximated technique that decreases the NOCPs to the quadratic programming problem (QPP). To our knowledge, there is no previous work focused on the use of the QPP based on the BPs basis for solving the NOCPs. Most approaches were used to solve various optimal control problems numerically, transform the original problem into the Lagrangian multipliers problem. Compare with the Lagrangian multipliers method, we do not need to solve the system of nonlinear algebraic equations which needs more computations and time. As is well-known, solving the system of nonlinear algebraic equations is also cumbersome, and sometimes, the solution may be unreliable. The proposed method in this paper transforms the original NOCPs into the QPP that can be solved by many subroutine algorithms and software with an alternative accurate. Using this method is easy and straightforward with more accuracy and less time and computations. This is because the new approximated problem only corresponds to the unknown BPs coefficient. This method only needs a small number of BPs basis to obtain very satisfactory and efficient results as well.

This paper is organized as follows: we first describe the fundamental formulation of BPs. Following, we explain the convergence of our method and, the operational matrices of BPs are introduced. Then, we state the wide class of NOCPs and introduce the proposed approach based on BPs. 
Eventually, we report numerical examples which show the validity, accuracy, efficiency, and applicability of the proposed method.

\section{Preliminaries}

In this section, some preliminaries of the BPs are given which will be used later

2.1. The properties of the BPs. The BPs of $m$-th degree on the interval $\left[0, t_{f}\right]$ is defined as follows: ([7])

$$
B_{i, m}(t)=\left(\begin{array}{c}
m \\
i
\end{array}\right) \frac{1}{\left(t_{f}\right)^{m}} t^{i}\left(t_{f}-t\right)^{m-i}, i=0,1, \ldots, m .
$$

Assume

$$
\phi(t)=\left[B_{0, m}(t) B_{1, m}(t) \ldots B_{m, m}(t)\right]^{T},
$$

Then $\phi(t)$ can be written as follows:

$$
\phi(t)=A T_{m}(t)
$$

where

$$
T_{m}(t)=\left[\begin{array}{llll}
1 t & t & \ldots & t^{m}
\end{array}\right]^{T}, \quad A=\{a(i, j)\}_{i, j=1}^{m+1},
$$

where $(\mathrm{i}, \mathrm{j})$-th entry of $A, a(i, j)$, defines as follow:

$$
a(i, j)= \begin{cases}(-1)^{j-i}\left(t_{f}\right)^{1-j}\left(\begin{array}{c}
m \\
i-1
\end{array}\right)\left(\begin{array}{c}
m-i+1 \\
j-i
\end{array}\right) & j \leq i \\
0 & j>i .\end{cases}
$$

Therefore $A$ is an $(m+1) \times(m+1)$ upper triangular matrix.

As is well-known, the BPs form a basis for the vector space of continuous polynomials which causes that any polynomial can be written as a linear combination of the BPs basis.

2.2. Function approximation. Presume $H=L^{2}\left[0, t_{f}\right]$ be Hilbert space with the inner product that is defined by $\langle f, g\rangle=\int_{0}^{t_{f}} f g d t$. Let $Y=$ $\operatorname{span}\left\{B_{0, m}, B_{1, m}, \cdots, B_{m, m}\right\}$, this set is a finite dimensional and closed subspace of $H$. Hence $Y$ is a complete subspace of $H$. Suppose $f$ is an arbitrary element in $H$, so $f$ has a unique best approximation out of $Y$, called it $y_{0} \in Y$, that is:

$$
\exists y_{0} \in Y, \quad \text { s.t. } \forall y \in Y, \quad\left\|f-y_{0}\right\|_{2} \leq\|f-y\|_{2},
$$

which $\quad\|f\|_{2}^{2}=\langle f, f\rangle$, (For more details see [23])

Since $y_{0} \in Y$, so there exist the unique coefficients $c_{0}, c_{1}, \ldots, c_{m}$ such that

$$
f(t) \simeq y_{0}=\sum_{i=0}^{m} c_{i} B_{i, m}=c^{T} \phi(t),
$$

where

$$
c^{T}=\left[c_{0}, c_{1}, \ldots, c_{m}\right]
$$


The vector $c^{T}$ can be obtained by:

$$
c^{T}\langle\phi, \phi\rangle=\langle f, \phi\rangle
$$

where

$$
\langle f, \phi\rangle=\int_{0}^{t_{f}} f \phi d t=\left[\left\langle f, B_{0, m}\right\rangle\left\langle f, B_{1, m}\right\rangle \ldots\left\langle f, B_{m, m}\right\rangle\right]
$$

and $\langle\phi, \phi\rangle$ is an $(m+1) \times(m+1)$ matrix and is called as dual matrix of $\phi$. Let

$$
Q=\langle\phi, \phi\rangle=\int_{0}^{t_{f}} \phi(t) \phi(t)^{T} d t
$$

then one can show that

$$
c^{T}=\left(\int_{0}^{t_{f}} f(t) \phi(t)^{T} d t\right) Q^{-1} .
$$

The next lemma shows that the error between the function and its best approximation reduces to zero as $m$ increases.

Lemma 2.1. ([12] ) Consider the real valued function $f$, where $f \in C^{m+1}\left[0, t_{f}\right]$, and

$Y=\operatorname{span}\left\{B_{0, m}, B_{1, m}, \cdots, B_{m, m}\right\}$. If $c^{T} \phi(t)$ be the best approximation $f$ out of $Y$ then the mean error bound is presented as follows:

$$
\left\|f-c^{T} \phi(t)\right\|_{2} \leq \frac{M t_{f}^{\frac{2 m+3}{2}}}{(m+1) ! \sqrt{2 m+3}}
$$

where $M=\max \left|f^{m+1}(\eta)\right|, \eta \in\left[0, t_{f}\right]$.

2.3. Operational matrices of the BPs. Here, we define operational matrices of BPs. Operational matrices of differentiation $D$, integration $P$, dual $Q$, and product $\widehat{C}$ of BPs are receptively defined by:

$$
\begin{aligned}
& \frac{d}{d t} \phi(t)=D \phi(t), \quad 0 \leq t \leq t_{f}, \\
& \int_{0}^{t} \phi(x) d x=P \phi(t), \quad 0 \leq t \leq t_{f}, \\
& Q=\int_{0}^{t_{f}} \phi(t) \phi(t)^{T} d t, \\
& C^{T} \phi(t) \phi(t)^{T}=\phi(t)^{T} \widehat{C},
\end{aligned}
$$

where the details of obtaining these matrices are given in [24]. 


\section{Problem statement}

Consider the following NOCPs,

$$
\begin{aligned}
\min J & =\frac{1}{2} \int_{0}^{t_{f}}\left[P x^{2}(t)+R u^{2}(t)\right] \mathbf{d} t, \\
\dot{x}(t) & =l(t) x(t)^{n}+s(t) u(t)^{m}+h(t) x(t) u(t) \\
& \quad+\alpha(t) x(t)+\beta(t) u(t) \\
x(0) & =x_{0}, \\
x\left(t_{f}\right) & =x_{f},
\end{aligned}
$$

where $x(t)$ is a state function, $u(t)$ is a control function. We denote the right hand side of equation(3.2) by $f(t, x, u)$. Thus $f$ is a continues nonlinear time varying function, where $f: A \times B \times\left[0, t_{f}\right] \longrightarrow R$, and $A \subseteq R, B \subseteq R$ are compact subset and must be chosen so that the system reach from initial state $x_{0}$ to final state $x_{f}$. Also $l(t), s(t), h(t), \alpha(t)$, and $\beta(t)$ are continuous functions which are the coefficients of $x(t)^{n}, u(t)^{m}, x(t) u(t), x(t)$, and $u(t)$, respectively, also $n$ and $m$ are integer number and $n, m \geq 0$. $P$ and $R$ are positive constants. The goal is finding the pair of $x(t)^{*}$ and $u(t)^{*}$ which minimizes the cost function of (3.1) and satisfies the nonlinear system (3.2)(3.4).

\section{Approximation solution based on BPs basis}

In this section, a new approach based on the BPs basis is presented to unravel the numerical solution of the NOCPs.

We approximate $x(t)$ and $u(t)$ by $X(t)$ and $U(t)$, respectively. Each $X(t)$ and $U(t)$ can be written in term of BPs as follows:

$$
\begin{aligned}
& x(t) \simeq X(t)=X^{T} \phi(t), \\
& u(t) \simeq U(t)=U^{T} \phi(t),
\end{aligned}
$$

where $\phi(t)=\left[\begin{array}{llll}B_{0, m}(t) & B_{1, m}(t) & \ldots & B_{m, m}(t)\end{array}\right]^{T}$ and $X^{T}=\left[\begin{array}{llll}\chi_{0} & \chi_{1} & \ldots & \chi_{m}\end{array}\right]$.

Furthermore since $l(t), s(t), h(t), \alpha(t)$, and $\beta(t)$ in general are not polynomials, we can approximate them by appropriate BPs as follows:

$$
\begin{aligned}
& l(t) \simeq L^{T} \phi(t), \\
& s(t) \simeq S^{T} \phi(t), \\
& h(t) \simeq H^{T} \phi(t), \\
& \alpha(t) \simeq A^{T} \phi(t), \\
& \beta(t) \simeq B^{T} \phi(t) .
\end{aligned}
$$

With using the operational matrix of product (2.12) ,we have:

$$
x^{2}(t)=X^{T} \phi(t) \phi(t)^{T} X=X^{T} \widehat{X} \phi(t),
$$


also

$$
x^{3}(t)=X^{T} \widehat{X} \phi(t) \phi(t)^{T} X=X^{T} \widehat{X}^{2} \phi(t) .
$$

By following the above procedure, we have

$$
x^{n}(t)=X^{T} \widehat{X}^{n-1} \phi(t)
$$

So

$$
l(t) x^{n}(t)=X^{T} \widehat{X}^{n-1} \phi(t) \phi(t)^{T} L=X^{T} \widehat{X}^{n-1} \widehat{L} \phi(t) .
$$

Similarly

$$
u^{m}(t)=U^{T} \widehat{U}^{m-1} \phi(t)
$$

and

$$
s(t) u(t)^{m}=U^{T} \widehat{U}^{m-1} \phi(t) \phi(t)^{T} S=U^{T} \widehat{U}^{m-1} \widehat{S} \phi(t) .
$$

Also, we have:

$$
\begin{aligned}
& x(t) u(t)=X^{T} \widehat{U} \phi(t), \\
& h(t) x(t) u(t)=X^{T} \widehat{U} \widehat{H} \phi(t) . \leq r,
\end{aligned}
$$

And finally we can write:

$$
\begin{aligned}
& \alpha(t) x(t)=X^{T} \widehat{A} \phi(t), \\
& \beta(t) u(t)=U^{T} \widehat{B} \phi(t) .
\end{aligned}
$$

With using the differentiation operational matrix (2.9), we have:

$$
\begin{aligned}
\dot{X}(t)=\frac{d}{d t} X(t) & =\frac{d}{d t}\left(X^{T} \phi(t)\right) \\
& =X^{T} \frac{d}{d t} \phi(t)=X^{T} D \phi(t) .
\end{aligned}
$$

Thus, for the nonlinear system of equations (3.2)-(3.4), with the above equations we obtain:

$$
\begin{gathered}
X^{T} D \phi(t)=X^{T} \widehat{X}^{n-1} \widehat{L} \phi(t)+U^{T} \widehat{U}^{m-1} \widehat{S} \phi(t) \\
+X^{T} \widehat{U} \widehat{H} \phi(t)+X^{T} \widehat{A}^{T} \phi(t)+U^{T} \widehat{B} \phi(t), \\
X^{T} \phi(0)=X_{0}, \quad X^{T} \phi\left(t_{f}\right)=X_{f} .
\end{gathered}
$$

With eliminating $\phi$ and putting the whole of terms on the left hand side of equalization (4.14), we obtain:

$$
\begin{aligned}
R_{1}= & X^{T} D-X^{T} \widehat{X}^{n-1} \widehat{L}-U^{T} \widehat{U}^{m-1} \widehat{S} \\
& -X^{T} \widehat{U} \widehat{H} X^{T} \widehat{A}^{T}-U^{T} \widehat{B} .
\end{aligned}
$$

Finally, we approximate the objective function (3.1) as follows:

$$
\begin{aligned}
& x^{2}(t)=X^{T} \phi(t) \phi(t)^{T} X, \\
& u^{2}(t)=U^{T} \phi(t) \phi(t)^{T} U,
\end{aligned}
$$


By substituting (4.15) into (3.1), we obtain:

$$
\begin{aligned}
R_{2}=\frac{1}{2} P X^{T} & {\left[\int_{0}^{t_{f}} \phi(t) \phi^{T}(t) d t\right] X } \\
& +\frac{1}{2} R U^{T}\left[\int_{0}^{t_{f}} \phi(t) \phi^{T}(t) d t\right] U .
\end{aligned}
$$

Then (3.1) can be rewritten as follows:

$$
R_{2}=\frac{1}{2} P X^{T} Q X+\frac{1}{2} R U^{T} Q U
$$

where as (2.11), the matrix $\mathrm{Q}$ is as follows:

$$
\int_{0}^{t_{f}} \phi(t) \phi^{T}(t) \mathrm{d} t=Q
$$

Also, we may recall that $Q$ and $P$ are positive definite matrices, so $R_{2}$ is a non-negative quadratic form. Now the NOCPs (3.1)- (3.4) can be reduced to the following nonlinear programming problem.

$$
\begin{array}{ll}
\min & M\left\|R_{1}\right\|^{2}+R_{2}, \\
\text { s.t. } & X^{T} \phi(0)=x_{0}, X^{T} \phi\left(t_{f}\right)=X_{f} .
\end{array}
$$

The new problem consists only the entries of the vectors $X$ and $U$. The norm we used in this optimization problem is the Euclidean norm, and $M$ is a penalty parameter. As we see, the approximation technique can reduce the basic dynamical systems to the QPP. Indeed, since $R_{1}$ is a linear equality, and $R_{2}$ is a non-negative quadratic form, the original problem can be reformulated as a QPP. The QPP (4.16) can be solved by many softwares, in this paper we used the package of Mathematica 10 to solve this problem.

Remark 4.1. This approach can be extended to a higher dimensional system. Let $r, q \in N$, then we can approximate $x(t)=\left[x_{1}(t) x_{2}(t) \cdots x_{r}(t)\right]^{T}$ and $u(t)=\left[u_{1}(t) u_{2}(t) \cdots u_{q}(t)\right]^{T}$ by

$$
\begin{aligned}
& X(t)=\left[\begin{array}{llll}
X_{1}(t) & X_{2}(t) & \cdots & X_{r}(t)
\end{array}\right]^{T} \\
& U(t)=\left[\begin{array}{llll}
U_{1}(t) & U_{2}(t) & \cdots & U_{q}(t)
\end{array}\right]^{T} .
\end{aligned}
$$

Each $X_{i}(t), 1 \leq i \leq r$ and $U_{j}(t), 1 \leq j \leq q$ can be written in terms of BPs, namely

$$
\begin{aligned}
& X_{i}(t)=X_{i}^{T} \phi(t) \\
& U_{j}(t)=U_{j}^{T} \phi(t),
\end{aligned}
$$

where $\phi(t)=\left[\begin{array}{llll}B_{0, m}(t) & B_{1, m}(t) & \ldots & B_{m, m}(t)\end{array}\right]^{T}$ and $X_{i}^{T}=\left[\begin{array}{llll}\mathcal{X}_{0 i} & \mathcal{X}_{1 i} & \ldots & \mathcal{X}_{m i}\end{array}\right]$. Similarly, with the same computation of (4.3)-(4.16), the original problem can be reformulated as QPP. 


\section{Numerical examples}

In this section, six examples are presented to illustrate the validity and efficiency of the proposed approach. The numerical results are programmed with the help of Mathematica 10 software.

Example 5.1. Consider the following time-varying optimal control problem [1].

$$
\begin{aligned}
J & =\frac{1}{2} \int_{0}^{1}\left(x^{2}(t)+u^{2}(t)\right) \mathrm{d} t, \\
\dot{x}(t) & =t x(t)+u(t), \\
x(0) & =1 .
\end{aligned}
$$

By choosing $m=6$, as the degree of BPs, we obtain the following solution

$$
\begin{gathered}
x(t)=(1-t)^{6}+5.03132(1-t)^{5} t+11.1579(1-t)^{4} t^{2} \\
+13.9881(1-t)^{3} t^{3}+10.5984(1-t)^{2} t^{4} \\
+4.60881(1-t) t^{5}+0.92174 t^{6} \\
u(t)=-0.968575(1-t)^{6}-4.80814(1-t)^{5} t \\
-9.54528(1-t)^{4} t^{2}-9.33825(1-t)^{3} t^{3} \\
-4.62694(1-t)^{2} t^{4}-0.918807(1-t) t^{5} \\
-0.0000824538 t^{6}
\end{gathered}
$$

The approximated objective function with $m=6$ is $J=0.484228$ and the value of objective function of Power series, Shifted Chebyshev (1 st kind), Shifted Chebyshev (2 nd kind), and Shifted Legendre with $m=6$ are $J=0.484072, J=0.484265, J=0.484265$ and $J=0.484262$, respectively. The graph of approximated solution $x(t)$ and $u(t)$ are plotted in the FIGURE 1. The Tables 1 and 2 demonstrate the numerical results for the state and the control variables, respectively, for the the mentioned methods and the proposed method in article with $m=6$.

Example 5.2. Consider the following NOCP [17].

$$
\begin{aligned}
J & =\frac{1}{2} \int_{0}^{1}\left(x^{2}(t)+u^{2}(t)\right) \mathrm{d} t, \\
\dot{x}(t) & =x(t) u(t)+3 u(t)-2 x(t), \\
x(0) & =5 .
\end{aligned}
$$


TABLE 1. Comparison of $x(t)$ using the different approaches for Example 5.1

\begin{tabular}{cccccc}
\hline $\mathrm{t}$ & $\begin{array}{c}\text { The proposed } \\
\text { method }\end{array}$ & $\begin{array}{c}\text { Power } \\
\text { series }\end{array}$ & $\begin{array}{c}\text { Shifted Chebyshev } \\
(2 \text { nd kind })\end{array}$ & $\begin{array}{c}\text { Shifted Chebyshev } \\
(1 \text { st kind })\end{array}$ & $\begin{array}{c}\text { Shifted } \\
\text { Legendre }\end{array}$ \\
\hline 0 & 1 & 0.999999 & 0.999999 & 1 & 1 \\
0.1 & 0.912841 & 0.9125870 & 0.9128372 & 0.9128436 & 0.9128402 \\
0.2 & 0.844075 & 0.8439083 & 0.8440804 & 0.8440875 & 0.8440838 \\
0.3 & 0.792566 & 0.7924710 & 0.7925795 & 0.7925848 & 0.7925822 \\
0.4 & 0.75766 & 0.7575558 & 0.7576795 & 0.7576830 & 0.7576814 \\
0.5 & 0.739162 & 0.7390190 & 0.7391855 & 0.7391888 & 0.7391874 \\
0.6 & 0.737388 & 07371867 & 0.7373666 & 0.7373711 & 0.7373689 \\
0.7 & 0.752962 & 0.7528404 & 0.7529964 & 0.7530020 & 0.7529991 \\
0.8 & 0.78739 & 0.7872928 & 0.7874314 & 0.7874368 & 0.7874340 \\
0.9 & 0.84268 & 0.8425564 & 0.8427275 & 0.8427314 & 0.8427295 \\
1 & 0.92174 & 0.9216030 & 0.9217929 & 0.9217992 & 0.9217963 \\
\hline
\end{tabular}

TABLE 2. Comparison of $u(t)$ using the different approach for Example 5.1

\begin{tabular}{cccccc}
\hline $\mathrm{t}$ & $\begin{array}{c}\text { The proposed } \\
\text { method }\end{array}$ & $\begin{array}{c}\text { Power } \\
\text { series }\end{array}$ & $\begin{array}{c}\text { Shifted Chebyshev } \\
(2 \text { nd kind })\end{array}$ & $\begin{array}{c}\text { Shifted Chebyshev } \\
(1 \text { st kind })\end{array}$ & $\begin{array}{c}\text { Shifted } \\
\text { Legendre }\end{array}$ \\
\hline 0 & -0.968575 & -0.9683481 & -0.9685254 & -0.9685323 & -0.9685288 \\
0.1 & -0.868473 & -0.8683751 & -0.8685338 & -0.8685402 & -0.8685370 \\
0.2 & -0.768625 & -0.7685019 & 0.7686403 & -0.7686461 & -0.7686431 \\
0.3 & -0.669056 & -0.6689425 & -0.6690605 & -0.6690654 & -0.6690630 \\
0.4 & -0.570052 & -0.5699850 & -0.5700840 & -0.5700883 & -0.5700862 \\
0.5 & -0.47197 & -0.4719342 & -0.4720162 & -0.4720199 & -0.4720180 \\
0.6 & -0.375096 & -0.3750550 & -0.3751203 & -0.3751233 & -0.3751218 \\
0.7 & -0.279572 & -0.2795159 & -0.2795623 & -0.2795649 & -0.2795635 \\
0.8 & -0.185361 & -0.1853325 & -0.1853572 & -0.1853591 & -0.1853581 \\
0.9 & -0.0922808 & -0.0923125 & -0.0923169 & -0.0923181 & -0.0923175 \\
1 & $-8.2454 e-05$ & $-1.74 e-08$ & $-5.25 e-09$ & $-8.99 e-08$ & $-2.69 e-09$ \\
\hline
\end{tabular}

By choosing $m=8$, we obtain the following solution

$$
\begin{aligned}
& x(t)=5(1-t)^{8}+2.49972(1-t)^{7} t+41.9064(1-t)^{6} t^{2} \\
& \quad+26.9588(1-t)^{5} t^{3}+4.55487(1-t)^{4} t^{4}+17.1269(1-t)^{3} t^{5} \\
& \quad+4.54265(1-t)^{2} t^{6}+0.667393(1-t) t^{7}+0.0801383 t^{8}, \\
& u(t)=-3.42941(1-t)^{8}-12.783(1-t)^{7} t+49.2549(1-t)^{6} t^{2} \\
& \quad-120.996(1-t)^{5} t^{3}+2.45668(1-t)^{4} t^{4}+24.8102(1-t)^{3} t^{5} \\
& \quad-6.88784(1-t)^{2} t^{6}-0.934623(1-t) t^{7}+0.0421773 t^{8} .
\end{aligned}
$$

The approximated objective function with $m=8$ is $J=1.47311$ and the value of objective function Block Plus function and Legendre polynomial with $m=10$ and $n=3$ in [17], is $J=1.4851$. The graph of approximated solution $x(t)$ and $u(t)$ are plotted in the FIGURE 2. 


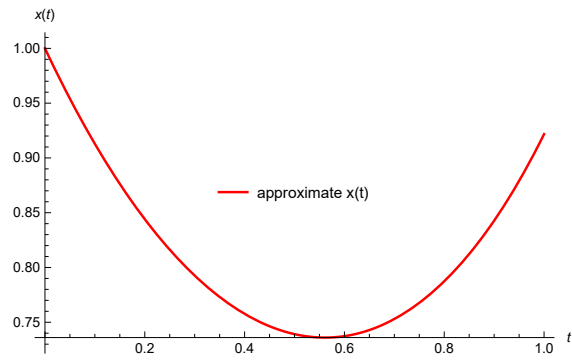

(A) $x(t)$

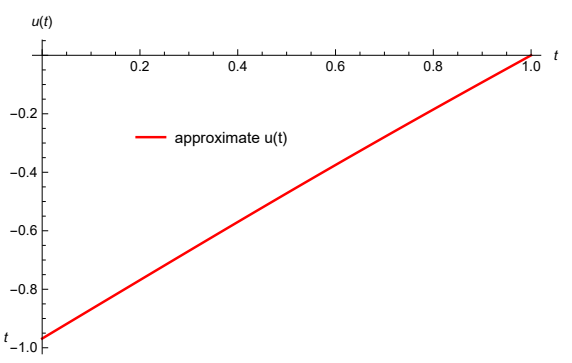

(B) $u(t)$

FigURE 1. The graph of approximated solution $x(t)$ and $u(t)$ for Example 5.1

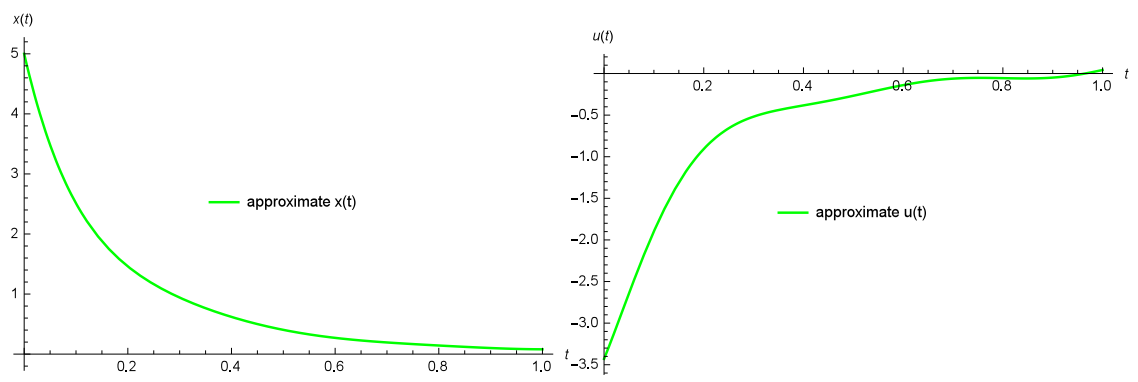

(A) $x(t)$

(B) $u(t)$

FiguRE 2. The graph of approximated solution $x(t)$ and $u(t)$ for Example 5.2

Example 5.3. Consider the following NOCP [18].

$$
\begin{aligned}
& \min J=\int_{0}^{1}\left[x_{1}^{2}(t)+x_{2}^{2}(t)\right] \mathrm{d} t, \\
& \dot{x_{1}}(t)=x_{2}(t), \\
& \dot{x_{2}}(t)=10 x_{1}^{3}(t)+u(t), \\
& x_{1}(0)=0 \quad x_{1}(1)=0.1 \text {, } \\
& x_{2}(0)=0 \quad x_{2}(1)=0.3 \text {. }
\end{aligned}
$$




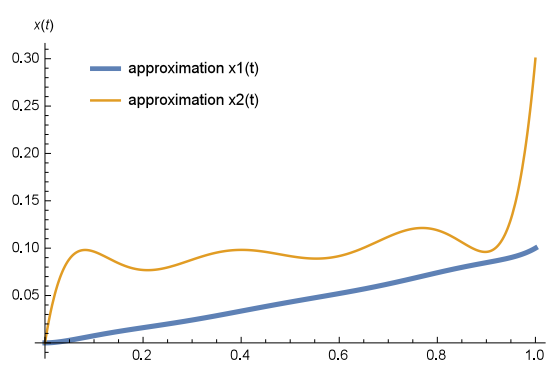

(A) $x_{1}(t)$ and $x_{2}(t)$

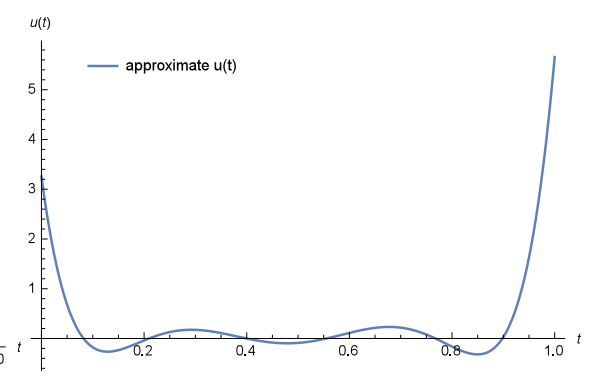

(B) $u(t)$

Figure 3. The graph of approximated solution $x_{1}(t), x_{2}(t)$ and $u(t)$ for Example 5.3

By choosing $m=8$, the approximate solutions of $x_{1}(t)$ and $x_{2}(t)$ with presented method are

$$
\begin{aligned}
x_{1}(t) & =2.57174 \times 10^{-7}(1-t)^{7} t+1.62669(1-t)^{6} t^{2} \\
- & 3.07993(1-t)^{5} t^{3}+11.2922(1-t)^{4} t^{4}-2.93251(1-t)^{3} t^{5} \\
\quad+ & 3.53359(1-t)^{2} t^{6}+0.5(1-t) t^{7}+0.1 t^{8}, \\
x_{2}(t)= & 3.25338(1-t)^{7} t-15.7466(1-t)^{6} t^{2}+41.5685(1-t)^{5} t^{3} \\
+ & 0.737089(1-t)^{4} t^{4}-29.8323(1-t)^{3} t^{5}+26.4319(1-t)^{2} t^{6} \\
& -3.26719(1-t) t^{7}+0.3 t^{8} .
\end{aligned}
$$

The value of cost function with the proposed method with $m=8$ is $J=$ 0.0136461 and it value obtained in [18] is $J=0.024$ also in [2] value of cost function is $J=0.0134817$. Thus with $m=8$, we achieved acceptable numerical results in comparison with what have been acquired in [18] and [2]. The graph of approximated solution $x_{1}(t), x_{2}(t)$ and $u(t)$ are plotted in the FIGURE 3.

Example 5.4. Consider the following NOCP [18].

$$
\begin{aligned}
\min J & =\int_{0}^{1} u^{2}(t) \mathrm{d} t, \\
\dot{x}(t) & =\frac{1}{2} x^{2}(t) \sin x(t)+u(t), \\
x(0) & =0 . \\
x(1) & =0.5 .
\end{aligned}
$$

The approximated cost function by the presented method with $m=8$ is $J=0.226676$, and the value of approximated cost function in [18] which has been solved this problem by Embedding method with $M=24, N=$ 1000, $M_{1}^{\prime}=6, M_{2}=8, L=10, P_{1}=24$ and $P_{2}=71$ is $j=0.2425$. So with $m=8$, we achieved satisfactory numerical results in comparison with what have been obtained in [18].The graph of approximated state and 


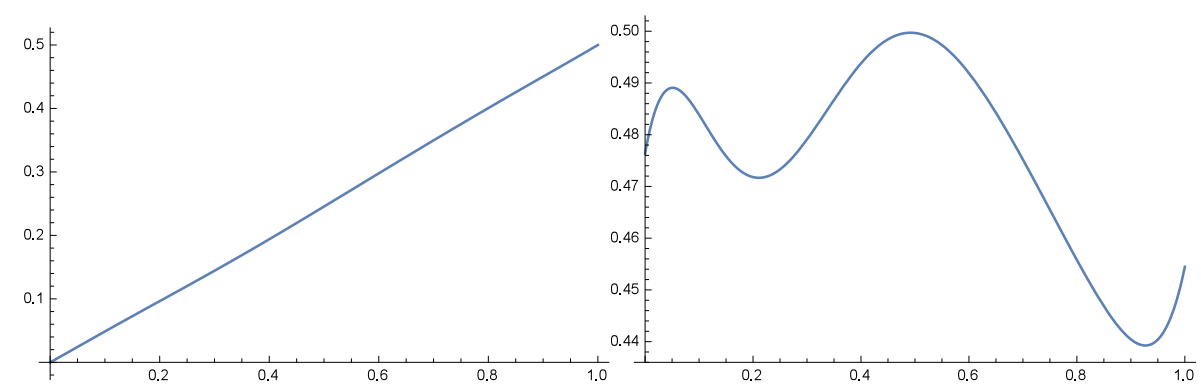

(A) $x(t)$

(B) $u(t)$

FIgURE 4. The graph of approximated solution $x(t)$ and $u(t)$ for Example 5.4

control are plotted in the FIGURE 4. Also the approximate solutions of $x(t)$ and $u(t)$ with presented method are

$$
\begin{aligned}
x( & (t)=0.47639(1-t)^{7} t+3.62987(1-t)^{6} t^{2} \\
& +8.86159(1-t)^{5} t^{3}+18.1152(1-t)^{4} t^{4}+17.0711(1-t)^{3} t^{5} \\
& +10.6956(1-t)^{2} t^{6}+3.48824(1-t) t^{7}+0.5 t^{8}, \\
u(t) & =0.476366(1-t)^{8}+4.40106(1-t)^{7} t+8.70318(1-t)^{6} t^{2} \\
& +32.6219(1-t)^{5} t^{3}+39.6488(1-t)^{4} t^{4}+23.7836(1-t)^{3} t^{5} \\
& +14.6908(1-t)^{2} t^{6}+3.13301(1-t) t^{7}+0.454464 t^{8} .
\end{aligned}
$$

Example 5.5. Consider the van der Pol oscillator problem [4].

$$
\begin{aligned}
\min J & =\frac{1}{2} \int_{0}^{5}\left(x_{1}^{2}(t)+x_{2}^{2}(t)+u^{2}(t)\right) \mathrm{d} t \\
\dot{x_{1}}(t) & =x_{2}(t) \\
\dot{x_{2}}(t) & =-x_{1}(t)+\left(1-x_{1}^{2}(t)\right) x_{2}(t)+u(t), \\
x_{1}(0) & =1 \\
x_{2}(0) & =0 .
\end{aligned}
$$

For solving this problem we choose $m=6$, the optimal value of the cost functional is obtained as $J=1.43168$. The objective value $J=1.438097$ has been achieved in [4] employing Quasilinearization and Discretization methods. In [8] the value of the objective function with second variation method is $J=1.433508$. Hence, our results with $m=6$ are in good agreement with the results shown in [4] and [8]. In addition, the approximate 


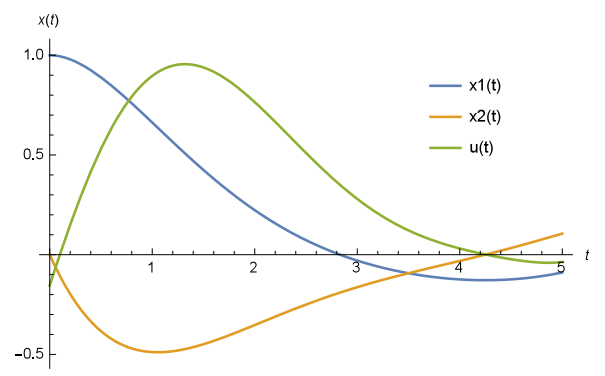

FIgURE 5. The graph of approximated $x_{1}(t), x_{2}(t)$ and $u(t)$ for Example 5.5

solutions of $x_{1}(t), x_{2}(t)$ and $u(t)$ with presented method are:

$$
\begin{gathered}
x_{1}(t)=\frac{(5-t)^{6}}{15625}+0.000383996(5-t)^{5} t \\
+0.0000314387(5-t)^{4} t^{2}+0.0000280601(5-t)^{3} t^{3} \\
-0.000135932(5-t)^{2} t^{4}-0.0000680602(5-t) t^{5} \\
\quad-5.71071 \times 10^{-6} t^{6}, \\
x_{2}(t)=-0.000371418(5-t)^{5} t-0.00037973(5-t)^{4} t^{2} \\
\quad-0.000133894(5-t)^{3} t^{3}-0.000139268(5-t)^{2} t^{4} \\
\quad-6.92806 \times 10^{-6}(5-t) t^{5}+6.75918 \times 10^{-6} t^{6}, \\
\text { and } \quad \begin{aligned}
& u(t)=-9.85503 \times 10^{-6}(5-t)^{6}+0.000448566(5-t)^{5} t \\
&+ 0.00200647(5-t)^{4} t^{2}-0.000645179(5-t)^{3} t^{3} \\
&+0.000327379(5-t)^{2} t^{4}-0.0000350843(5-t) t^{5} \\
& \quad-2.3217 \times 10^{-6} t^{6} .
\end{aligned}
\end{gathered}
$$

The figures of $x_{1}(t), x_{2}(t)$ and $u(t)$ are shown in the FIGURE 5 .

Example 5.6. Consider the following optimal control problem [19].

$$
\begin{aligned}
J & =\frac{1}{2} \int_{0}^{1}\left(x^{2}(t)+u^{2}(t)\right) \mathrm{d} t, \\
\dot{x}(t) & =-x(t)+u(t), \\
x(0) & =1, \\
x(1) & =0.5 .
\end{aligned}
$$

The exact solution is

$$
\begin{aligned}
& x(t)=0.129195 e^{\sqrt{2} t}-0.129195 e^{-\sqrt{2} t} \\
& u(t)=0.311903 e^{\sqrt{2} t}+0.0535141 e^{-\sqrt{2} t}
\end{aligned}
$$


By choosing $m=4$, the approximated solution is

$$
\begin{gathered}
x(t)=0.364339(1-t)^{3} t+1.10357(1-t)^{2} t^{2} \\
+1.20525(1-t) t^{3}+0.5 t^{4}, \\
u(t)=0.364281(1-t)^{4}+1.84252(1-t)^{3} t \\
+3.62578(1-t)^{2} t^{2}+3.40818(1-t) t^{3} \\
+1.29461 t^{4} .
\end{gathered}
$$

The Tables 3 and 4 demonstrate approximated solution, exact Solution and error between them for $m=4$. The graph of approximated solution and exact solution $x(t)$ and $u(t)$ are plotted in the FIGURE 6 .

TABLE 3. Comparison between of $x(t)$ exact and approximated solution for Example 5.6

\begin{tabular}{cccc}
\hline$t$ & $\begin{array}{c}\text { Approximated solution } \\
\text { with } m=4\end{array}$ & $\begin{array}{c}\text { Exact } \\
\text { solution }\end{array}$ & $\begin{array}{c}\text { Error between exact } \\
\text { and approximated solution }\end{array}$ \\
\hline 0 & 0 & 0 & 0 \\
0.1 & 0.036634 & 0.0366638 & $-0.4 e^{-5}$ \\
0.2 & 0.0740734 & 0.0740621 & $1.13 e^{-5}$ \\
0.3 & 0.112987 & 0.112944 & $4.3 e^{-5}$ \\
0.4 & 0.154126 & 0.154089 & $3.7 e^{-5}$ \\
0.5 & 0.198323 & 0.19832 & $0.3 e^{-5}$ \\
0.6 & 0.24649 & 0.246525 & $-3.5 e^{-5}$ \\
0.7 & 0.299624 & 0.299668 & $-4.4 e^{-5}$ \\
0.8 & 0.358801 & 0.358815 & $-1.4 e^{-5}$ \\
0.9 & 0.42518 & 0.42515 & $0.3 e^{-5}$ \\
1 & 0.5 & 0.5 & 0 \\
\hline
\end{tabular}

TABle 4. Comparison between of $u(t)$ exact and approximated solution for Example 5.6

\begin{tabular}{cccc}
\hline$t$ & $\begin{array}{c}\text { Approximated solution } \\
\text { with } m=4\end{array}$ & $\begin{array}{c}\text { Exact } \\
\text { solution }\end{array}$ & $\begin{array}{c}\text { Error between exact } \\
\text { and approximated solution }\end{array}$ \\
\hline 0 & 0.364281 & 0.0365417 & $-1.1 e^{-3}$ \\
0.1 & 0.40589 & 0.405741 & $1.49 e^{-4}$ \\
0.2 & 0.454587 & 0.454193 & $3.94 e^{-4}$ \\
0.3 & 0.511857 & 0.511745 & $1.12 e^{-4}$ \\
0.4 & 0.579265 & 0.579548 & $-2.83 e^{-4}$ \\
0.5 & 0.65846 & 0.658961 & $-5.01 e^{-4}$ \\
0.6 & 0.751171 & 0.751576 & $-4.05 e^{-4}$ \\
0.7 & 0.859209 & 0.859247 & $-3.8 e^{-5}$ \\
0.8 & 0.984465 & 0.984132 & $3.33 e^{-4}$ \\
0.9 & 1.12891 & 1.12873 & $1.8 e^{-4}$ \\
1 & 1.29461 & 1.29595 & $-1.3 e^{-3}$ \\
\hline
\end{tabular}




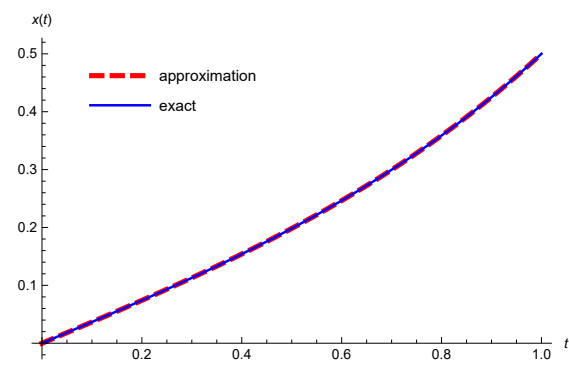

(A) $x(t)$

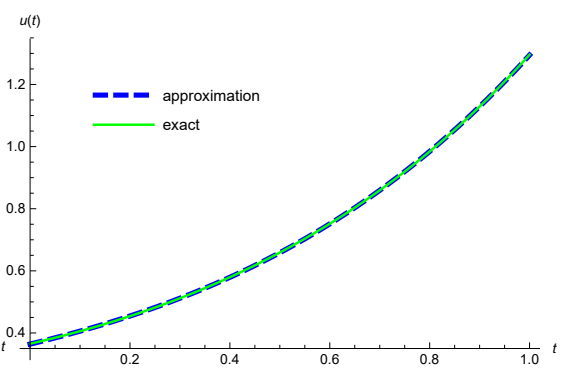

(B) $u(t)$

FIGURE 6. The graph of approximated and exact solution $x(t)$ and $u(t)$ for Example 5.6

\section{Conclusions}

In this paper, the operational matrices of integration, differentiation, and product for BPs are introduced. Also, an upper bound for the error of approximation is given. The presented upper bound of error demonstrates convergent to the exact solution when a degree of the BPs tends to infinity. The BPs are used to solve NOCPs. The problem has been reduced to solve a QPP which can be solved by many softwares with alternative accuracy and without solving any large system of nonlinear algebraic equations. Comparing with other methods, for instance, Quasilinearization, Linearization, and Discretization, the proposed method is general with less time and calculations, easy to implement, and yields accurate results. However, the upper bound of error proves the convergence of this method. Authors understand that this technique for large $m$ does not work effectively, since the error computing by computer, arises with increasing $m$. But we found that one can easily achieve satisfactory results with some small $m$, such as $m=6,7,8$, in comparison with other methods. The illustrative examples demonstrate that the presented method is valid and effective.

\section{REFERENCES}

[1] O. P. Agrawal, General formulation for the numarical solution of optimal control problems, Int. J. Control. 20(2) (1989) 627-638.

[2] K. P. Badakhshan and A. V. Kamyad, Numerical solution of nonlinear optimal control problems using nolinear programming, AMC. 187 (2007) 1511-1519.

[3] M. V. Basin, P. Shi And D. C. Alvarez, Central suboptimal $h_{\infty}$ control design for nonlinear polynomial systems, Int. J. Systems Science. 42 (2011) 801-808.

[4] G. Bashein And M. Enns, Computation of optimal control by a method combining quasi-linearization and quadratic programming, Int. J. Control. 16(1) (1972) 177-187.

[5] M. Behroozifar, S. A. Yousefi And A. N. Ranjbar, Numerical solution of optimal control of time-varying Singular Systems via operational Matrices, Int. J. Engineering. 27(4) (2014) 523-532.

[6] M. I. Bhatti, Solution of fractional harmonic oscillator in a fractional B-poly basis, PTS. 2(2) (2014), 8-13. 
[7] M. I. Bhatti And P. Bracken, Solutions of differential equations in a Bernstein polynomial basis, J. Comput. Appl. Math. 205 (2007) 272-280.

[8] T. Bullock And G. Franklin, A second order feedback method for optimal control computations, IEEE Trans. Autom. Control. 12(6) (1967) 666-673.

[9] M. Dehghan, S. A. Yousefi and K. Rashedi, Ritz-Galerkin method for solving an inverse heat conduction problem with a nonlinear source term via Bernstein multiscaling functions and cubic B-spline function. Inverse Probl. Sci. En. 21(3) (2013) $500-523$.

[10] M. R. Eslahchi and M. Dehghan, Application of Taylor series in obtaining the orthogonal operational matrix, Comput. Math. with Appl. 61(9) (2011) 2596-2604.

[11] H. JADDU, Direct solution of nonlinear optimal control problems using quasilinearization and Chebyshev ploynomial, J. Franklin Inst. 339 (2002) 479-498.

[12] N. Ghaderi AND M. H. FARAhi, The numerical solution of some optimal control systems with constant and pantograph delays via Bernstein polynomials. IJMSI, 15(2) (2020) 163-181.

[13] M. H. Gonzaleza And M. V. Basina, Discrete time optimal control for stochastic nonlinear polynomial systems. Int. J. Gen. Syst., 43(3-4) (2014) 359-371.

[14] A. Kayedi-Bardeh, M. R. Eslahchi and M. Dehghan, A method for obtaining the operational matrix of the fractional Jacobi functions and applications. J. Vib. Control, 20(5) (2014) 736-748.

[15] T. T. Lee And Y. F. Chang, Parameter and optimal control of nonlinear systems via general orothogonal ploynomials. Analysis, Int. J. Control, 4(44) (1986) 10891102.

[16] S. Mashayekhi, Y. Ordokhani and Y. Razzaghi, Hybrid function approach for nonlinear constrained optimal control problem. Commun Nonlinear Sci Numer Simulat., 17 (2012) 1831-1843.

[17] B. M. MOHAN AND S. K. KAR, Optimal control of nonlinear systems via orthogonal function, Automation and Signals(ICEAS), Bhubaneswar, India., 28(4) (1994) 28 30 .

[18] J. E. Rubio Control and optimization; the linear treatment of nonlinear problems, Manchester University Press, (1985).

[19] E. R. Pinch Optimal Control and the calculus of variation, Oxford University Press, (1993).

[20] A. Saadatmandi And M. Dehghan, A new operational matrix for solving fractionalorder differential equations. Comput. Math. with Appl. 59(3), (2010), 1326-1336.

[21] W. ShiEnyu, Convergence of Block pluse series approximation for optimal control problem, Int. J. Systems Science. 21(7) (1990) 1335-1368.

[22] J. Vlassenbroeck and R. V. Dooren, A Chebyshev technique for solving nonlinear optimal control problems, IEEE Trans. Automatic Control. 33(4) (1988) 333-340.

[23] G. A. Watson, Approximation theory and numerical methods. John Wiley \& Sons, (1980).

[24] S. A. Yousefi And M. Behroozifar, Operational matrices of Bernstein polynomials and their applications, Int. J. Syst. Sci. 41, (2010), 709-716.

[25] S. A. Yousefi, M. Behroozifar and M. Dehghan, The operational matrices of Bernstein polynomial for solving the parabolic equation subject to specification of the mass, J. Comput. Appl. Math 235, (2011), 5272-5283.

[26] S. A. Yousefi, M. Behroozifar and M. Dehghan, Numerical solution of the nonlinear age-structured population models by using the operational matrices of Bernstein polynomials, Appl. Math Model. 36(3), (2012), 945-963. 
(Najmeh Ghaderi) Department of Applied Mathematics, Factually of Mathematical Sciences, Ferdowsi University of Mashhad, Mashhad, Iran

Email address: najmeh.ghaderi@mail.um.ac.ir

(Mohammad Hadi Farahi) Department of Applied Mathematics, Factually of Mathematical Sciences, Ferdowsi University of Mashhad, Mashhad, Iran, and The Center of Excellence on Modelling and Control Systems (CEMCS), MASHHAD, IRAN

Email address: farahi@math.um.ac.ir 